\title{
Mycoplasma genitalium Detected by Transcription-Mediated Amplification Is Associated With Chlamydia trachomatis in Adolescent Women
}

\author{
Jill S. Huppert, MD, MPH${ }^{\star}$, Joel E. Mortensen, $\mathrm{PhD}^{\dagger}$, Jennifer L. Reed, MD ${ }^{\ddagger}$, Jessica A. \\ Kahn, MD, MPH ${ }^{\star}$, Kimberly D. Rich, MPH ${ }^{\S}$, and Marcia M. Hobbs, PhD $\S, \|$ \\ "Division of Adolescent Medicine, Cincinnati Children's Hospital Medical Center and the \\ University of Cincinnati College of Medicine, Cincinnati, Ohio \\ tLaboratory Medicine, Cincinnati Children's Hospital Medical Center and the University of \\ Cincinnati College of Medicine, Cincinnati, Ohio \\ ‡Emergency Medicine, Cincinnati Children's Hospital Medical Center and the University of \\ Cincinnati College of Medicine, Cincinnati, Ohio \\ §Department of Medicine, University of North Carolina, Chapel Hill, North Carolina \\ "Department of Microbiology and Immunology, University of North Carolina, Chapel Hill, North \\ Carolina
}

\begin{abstract}
Objectives-The clinical significance of Mycoplasma genitalium (MG) infection in adolescent women is poorly understood. We compared the prevalence of MG with that of other sexually transmitted organisms such as Chlamydia trachomatis (CT), Neisseria gonorrhoeae (NG), and Trichomonas vaginalis (TV) and assessed the associations of MG with sexual behaviors, genitourinary symptoms, physical and laboratory findings.
\end{abstract}

Study Design-Women aged 14 to 21 years $(n=331)$ were recruited from an urban medical center. The subjects' sexual behaviors, genitourinary symptoms, and physical findings were recorded. Endocervical swabs were collected for CT and NG testing and vaginal swabs for wet mount, Gram stain, TV and MG testing. MG infection was identified by nucleic acid amplification using a transcription-mediated amplification assay.

\begin{abstract}
Results-MG was detected in 74 (22.4\%), CT in 79 (24.4\%), TV in 60 (18.2\%), and NG in 35 $(10.7 \%)$ subjects. MG infection was not associated with vaginal symptoms, physical evidence of cervicitis, or findings on wet mount or Gram stain. In logistic regression, variables positively associated with MG were current CT [odds ratio (OR), 2.3; 95\% confidence interval (CI), 1.4-4.4] and recent sexual contact ( $\leq 7$ days) (OR, 2.0; CI, 1.1-3.2). Dysuria (OR, 0.44; CI, 0.2-0.96) and use of hormonal contraception (OR, 0.55 ; CI, 0.3-1.0) were negatively associated with MG infection.
\end{abstract}

Conclusion-In adolescent women, MG infection was as common as chlamydial infection and trichomoniasis and more common than gonorrhea. MG was associated with $\mathrm{CT}$ and recent sexual contact but not with vaginal symptoms or signs of cervicitis.

Copyright @ 2008, American Sexually Transmitted Diseases Association All rights reserved.

Correspondence: Jill S. Huppert, MD, MPH, Cincinnati Children's Hospital Medical Center, Division of Adolescent Medicine, 3333 Burnet Avenue, ML 4000, Cincinnati, OH 45229-3039. jill.huppert@ cchmc.org. 
ADOLESCENT WOMEN FREQUENTLY COMPLAIN of genitourinary symptoms and are at high risk for sexually transmitted infections (STI) with organisms such as Chlamydia trachomatis (CT), Neisseria gonorrhoeae (NG), and Trichomonas vaginalis (TV). However, clinicians often cannot establish a definitive diagnosis for vaginitis or cervicitis. ${ }^{1}$ In addition, symptoms sometimes persist despite treatment. Infection with a sexually transmitted organism that is difficult to culture, such as Mycoplasma genitalium (MG), may explain genitourinary symptoms or poor response to treatment of other STI. Yet, little is known about the prevalence or significance of MG in adolescent women who are at high risk for STI.

MG has been identified as an etiologic agent of urethritis in men. ${ }^{2}$ However, in women, the results from studies that examined the associations between MG and genitourinary symptoms or signs are inconsistent. In one study using stored specimens that were obtained in the 1980s from women attending a sexually transmitted disease clinic, the odds of having mucopurulent cervicitis (MPC) among MG-positive women as detected by polymerase chain reaction (PCR) were threefold higher than among women without MG. ${ }^{3}$ In another study using detailed symptom constructs, MG detected by culture or PCR was associated with abnormal vaginal discharge but not MPC. ${ }^{4}$ In other studies, neither clinical signs nor patient symptoms were associated with MG infection.,

Recently, a transcription-mediated research assay (TMA) has been shown to be highly sensitive and specific for the detection of MG and to perform better on vaginal swabs than cervical or urine samples. ${ }^{7}$ The advent of nucleic acid amplification testing provides opportunities to more accurately determine the prevalence of MG in adolescent women and to determine whether, in this population, MG is associated with genitourinary symptoms, signs, or other STI. In addition, it may be important to identify clinical or laboratory findings that predict which patients may benefit from targeted MG testing, since widespread screening for MG is unlikely to occur in the near future. If strong associations were found between MG and other STI, presumptive treatment for MG may even be indicated, echoing the approach used in the past when, in the absence of sensitive tests for CT, patients with gonorrhea were treated presumptively for chlamydial infections.

The objectives of the current study were to describe the prevalence of MG in sexually active adolescent women at high risk for STI and to explore the associations of MG with sexual behaviors, genitourinary symptoms, physical and laboratory findings, and other STI.

\section{Subjects, Materials, and Methods}

\section{Subjects}

Female adolescents aged 14 to 21 years were recruited from an urban, hospital-based Teen Health Center (THC) or emergency department (ED) between July 2004 and May 2006 to participate in a cross-sectional STI screening study. The full study has been described previously. ${ }^{8}$ Inclusion criteria were a history of heterosexual contact in the last 6 months and reporting either genitourinary symptoms or a risk for STI (report of $>1$ sexual partner in the last 3 months, a new sexual partner in the last 3 months, or inconsistent condom use). Subjects signed informed consent, and the institutional review board approved the study with a waiver of the requirement for parental permission for participants $<18$ years of age.

Each subject was interviewed confidentially by a trained research assistant. The interviewer collected demographic variables and assessed self-reported sexual behaviors (condom use, contraceptive use, number of sexual partners in the last 90 days, date of last sexual intercourse) and elicited current genitourinary symptoms (vaginal itching or discharge, dysuria, abnormal bleeding, and pelvic pain) using a structured data-collection instrument. A 
pelvic exam was performed by a trained clinician, and physical findings during the pelvic exam (abnormal vaginal discharge, mucopurulent cervical discharge, cervical friability, bleeding, or pelvic tenderness) were recorded on the data collection form. During the speculum portion of the pelvic exam, the clinician obtained swabs from the endocervix and the vaginal fornices for laboratory testing.

\section{Laboratory Methods}

Endocervical swabs were tested for CT using a strand displacement assay (BD ProbeTec ET®, Becton Dickinson, Sparks, MD), and for NG using either culture or the strand displacement assay at the clinician's discretion.

For subjects recruited from the THC, a complete wet mount evaluation was performed in the on-site laboratory by an experienced clinician. For subjects recruited from the ED, vaginal swabs were transported to the clinical laboratory, where a Gram stain was examined. Wet mount or Gram stain slides were read at $400 \times$ magnification to quantitate white blood cells (WBCs) and the proportion of epithelial cells that were clue cells, which were categorized as $\unlhd 10$ versus $>10 \mathrm{WBCs}$ and $\_0 \%$ versus $>20 \%$ clue cells per field, respectively. Yeast forms seen on wet mount or Gram stain were considered a positive result for yeast.

After subject recruitment was complete, dry vaginal swabs that had been stored at $-80^{\circ} \mathrm{C}$ were processed in APTIMA $®$ swab transport medium and were tested for TV and MG using TMA assays performed on a DTS 400 instrumentation system (Gen- Probe, San Diego, CA). The TV assay used $T$. vaginalis analyte specific reagents with the APTIMA® General Purpose Reagents (GPR) as described previously. ${ }^{8}$

For MG TMA, $400 \mu \mathrm{L}$ of processed vaginal swab specimen was tested as previously described. ${ }^{7}$ Briefly, oligonucleotides specific for MG 16S rRNA were used for target capture and amplification using the APTIMA ${ }^{\circledR}$ GPR. Purified MG total nucleic acids and sterile water were used as positive and negative controls, respectively.

\section{Definitions of Outcomes}

Clinical samples for CT and NG were deemed positive if the corresponding tests were positive. TMA results $>30,000$ relative light units (RLU) were considered positive for TV. MG samples with initial results $>50,000$ RLU were retested from the same processed specimen, and specimens with repeat results $>50,000 \mathrm{RLU}$ were considered positive for MG. One sample was positive on initial testing but negative on repeat testing and was classified as negative. All samples with initial results $<50,000$ RLU were considered negative for MG.

MPC was defined as the presence of purulent or yellow discharge at the cervical os. Cervical friability was defined as easy bleeding with application of a swab to the cervix. Clinical cervicitis was defined when the provider marked either "MPC" or "cervical friability" on the data collection form. Vaginal symptoms included any chief complaint or elicited patient report of vaginal itching or discharge. Any genitourinary symptoms were recorded as present if the interview revealed a patient report of vaginal symptoms, dysuria, abnormal vaginal bleeding, or pelvic pain. Hormonal contraception included the use of combined oral contraceptive pills, the contraceptive patch or depot medroxyprogesterone acetate.

\section{Statistical Analyses}

Dichotomous variables were compared with MG status using $X^{2}$ tests, and continuous variables were compared using Student $t$ test. Analyses were performed on the full data set and then repeated on 4 subsets: those without CT, those without any other STI, those 
without vaginal infections (TV or yeast), and those without infections related to cervicitis (CT and NG).

Because we used 2 methods to ascertain clue cells and WBCs in vaginal fluid (wet mount or Gram stain), we stratified the sample to see if associations with MG varied by this method. We found no significant associations between MG and wet mount or Gram stain findings; therefore, we combined the data for wet mount and Gram stain results.

Logistic regression models were used to determine which variables were independently associated with MG infection. Variables that demonstrated an association with MG at $P<0.1$ in the bivarate analyses were included in the multivariable models. Because several risk behavior variables were highly correlated, the impact of each of these variables was tested by comparing the full and reduced models using a likelihood ratio test. The most parsimonious model is presented.

\section{Results}

Three hundred sixty-nine subjects were recruited; 331 with samples available for MG testing comprised the study sample. MG was detected in 74 (22.4\%) subjects, CT in 79 (24.4\%), TV in $60(18.2 \%)$, and NG in $35(10.7 \%)$ subjects. The mean age of participants was 17.7 years (range, 14-21); other demographics and subject history are displayed in Table 1. Those with MG were more likely to report sex in the last 7 days $(58.1 \%$ vs. $42.8 \%, P=0.02)$ and $>1$ sexual partner in the last 3 months $(35.2 \%$ vs. $23.4 \%, P=0.04)$ than those without MG. In addition, those with MG were slightly less likely to report using hormonal contraception at last intercourse (25.7\% vs. $37.7 \%, P=0.055)$. Although in this sample, those age 14 to 17 were more likely to have CT than those 18 to $21(29.2 \%$ vs. $18.0 \%, P=$ 0.02), younger age was not associated with MG, NG, or TV.

We evaluated symptoms reported by subjects and signs documented by the provider individually and in groups and found no differences between those with or without MG infection (Table 2). Wet mount and Gram stain results also were not associated with MG, whether analyzed together or separately. There was a trend toward greater dysuria and uterine/adnexal tenderness in those without MG infection that was not significant in bivariate analyses.

Adolescent women with MG had higher prevalence of CT, NG, and TV; this difference was statistically significant only for CT (Table 2). Thus, women with MG were more likely to be coinfected with CT than women without MG (37.5\% vs. $20.0 \%, P=0.003)$. More than half of young women with MG had another STI, compared with about a-third of those without MG $(P=0.003)$. However, no other clinical signs or patient-reported symptoms were significantly associated with $\mathrm{MG}$ infection.

A logistic regression model was developed to assess the independent contribution of variables associated with MG infection (Table 3). Both current CT infection and recent sexual intercourse (within the last 7 days) were associated with a twofold increase in the odds of MG infection. Dysuria and use of hormonal contraception at last sexual contact were associated with decreased odds of MG infection. A report of multiple partners was not significant and was dropped from the final model.

We assessed whether an MG infection could explain any patient- reported symptoms or clinical signs that were not explained by another infection. Thus, we repeated the analyses on the subsets of subjects that were negative for CT $(n=245)$, negative for CT and NG $(n=$ 226), negative for all other STI $(n=202)$, and negative for other vaginal infections (TV and 
yeast $)(\mathrm{n}=208)$. We identified no significant associations between MG status and symptoms or signs in any of these subsets (data not shown).

In contrast to our findings with MG, clinical cervicitis was associated with CT infection. Women with cervicitis were more likely to have CT than those without cervicitis (36.9\% vs. $19.8 \%, P=0.02)$. NG prevalence did not differ between those with and without cervicitis $(10.7 \%$ vs. $9.7 \%)$. In the absence of CT infection $(\mathrm{n}=235)$, MG prevalence did not differ between those with and without cervicitis ( $15.1 \%$ vs. $19.2 \%, P=0.5)$. In those with CT infection ( $\mathrm{n}=76) \mathrm{MG}$ prevalence was slightly lower in those with cervicitis compared to those without ( $25.8 \%$ vs. $40.2 \%, P=0.2$ ), but this difference did not reach statistical significance.

Of note, $44 \%$ of women with vaginal symptoms and $58 \%$ of those with clinical cervicitis had no documented infection. It is possible that bacterial vaginosis or other noninfectious conditions could account for some of these symptoms and signs.

\section{Discussion}

In this sample of adolescent women, we documented a high prevalence of MG (22\%) that was comparable to the prevalence of CT (24\%) and TV (18\%) and was higher than that of NG. Other studies have reported a similar prevalence of MG in women: $15 \%$ in a Seattle STD clinic (18-27-year-old women $)^{7} ; 19 \%$ in a Baltimore STD clinic of adult women ${ }^{9}$; and $16 \%$ among 18- to 35-year-old Nairobi sex workers. ${ }^{5}$ In contrast, the prevalence of MG in STD clinics in Sweden was $6.3 \%,{ }^{10}$ and up to $4.1 \%$ in a longitudinal, nonclinic based sample of adolescents in Indianapolis. ${ }^{6}$

The higher prevalence of MG we report here in adolescent women seeking care is not surprising, since we purposefully recruited a sample at high risk for all STI. Elicited genitourinary symptoms were common (any symptom present in 89\%) and overlapped considerably with other risks for STI (shown in Table 1), with 62\% reporting a history of STI, 52\% reporting no condom use at last sexual contact, and $26 \%$ reporting a new partner in the last 3 months.

In our sample, there were a large number of women aged 14 to 17 who were at higher risk for CT than those aged 18 to 21 . However, the MG prevalence in these younger women $(21.3 \%)$ was similar to that of women aged 18 to $21(23.7 \%)$. One explanation may be that unlike CT, MG does not have a strong affinity for the immature adolescent cervix.

Regardless of age, young women in our cross-sectional study were frequently coinfected with MG and CT. Similar results were seen in 2 longitudinal studies. In the cohort of Nairobi sex workers, incident CT increased the hazard of acquiring MG 2.5-fold, and in adolescents, the odds of MG in the presence of CT were 1.9.5,6 These results are similar to our findings. This relationship may have implications for understanding the pathogenicity and possible treatment options for MG.

In this sample of young women at risk for STI, MG infection was not independently correlated with higher-risk sexual behaviors such as inconsistent condom use, new partners, or multiple sexual partners. MG prevalence was greater with shorter interval since last sexual contact. This may represent spontaneous clearance of infection or exposure to adequate antibiotic therapy. In a longitudinal study, $70 \%$ to $90 \%$ of MG infections resolved within 3 to 7 months without treatment. ${ }^{5}$ The protective effect of hormonal contraception that we found may be because of changes in the vaginal ecosystem related to the hormones themselves. Alternatively, this variable may be a marker for an unmeasured behavior, such as being in a long-term monogamous relationship, which may decrease the risk of MG. 
We did not identify any clinical findings that were reliably associated with MG infection. This is disappointing in light of reports that a clinical finding of vaginal leukocyte count (>10 WBC/high powered field) is predictive of infections with CT, NG, and TV. ${ }^{11,12} \mathrm{In}$ addition, we were unable to attribute clinical cervicitis to MG infection, even in those without CT or NG. Results from other studies are conflicting, and may be because of differences in the populations studied, the methods used for MG detection, or the definitions used for cervicitis. Three of six recent studies showed an association between MG and cervicitis. ${ }^{3,10,13}$ In these 3 studies, the populations were adult STD clinic attendees, many reported vaginal symptoms, and the prevalence of $\mathrm{MG}$ ranged from $6 \%$ to $26 \%$. MG was detected by in-house PCR from cervical samples, some of which had been frozen, ${ }^{3}$ while others were performed at the time of evaluation. Cervicitis was variably defined as cervical mucopus, friability or $>30$ polymorphonuclear leukocytes $/ 1000 \times$ field on Gram stain. In 2 longitudinal studies ${ }^{5,6}$ and 2 cross-sectional studies ${ }^{4,14}$ without demonstrated associations between MG and clinical cervicitis, MG was defined by culture or PCR; cervicitis was defined as any of 4 clinical signs or the presence of more polymorphonuclear leukocytes than epithelial cells on a wet smear of vaginal secretions. Given this wide variation in definitions of both MG infection and clinical cervicitis, it is not surprising that statistical measures of association are inconsistent.

In our adolescent sample, MG infection was not correlated with signs or symptoms of vaginitis. This is consistent with findings from a longitudinal study of adolescents by Tosh et al. ${ }^{6}$ However, we did not differentiate between chief complaint and elicited symptoms, and our attempt to standardize recording of symptoms may have encouraged overreporting in these adolescent women. In addition, both our study and that of Tosh et al. relied upon patient self-report and did not include the patient's perception of whether the symptoms were abnormal or bothersome, as has been described by some authors. ${ }^{15}$

The negative association with dysuria is perplexing, as others have found no association between MG and urinary symptoms in adult women. ${ }^{10}$ Our findings may be related to the adolescent's inability to describe her genitourinary symptoms accurately. In light of the evidence that MG is associated with symptomatic urethritis in studies of men, 2,10 asymptomatic infection in women should not be discounted.

This study has the advantage of a large sample of adolescent females and sensitive laboratory methods to detect infection with the 4 organisms studied: CT, NG, TV, and MG. The study is limited by the cross-sectional design, which does not allow us to examine temporal relationships or the risk of consequences, such as pelvic inflammatory disease or HIV acquisition. Another limitation is that subjects were recruited for their known STI risks; thus the prevalence in this study sample will likely differ from other populations with lower risks. Additionally, we relied upon patient reporting of symptoms and clinician reporting of signs and physical findings. The use of multiple clinicians to assess cervicitis may have resulted in some misclassification that contributed to our observed lack of association between MG and cervicitis.

The role of MG as a pathogen should be further explored. It is well accepted that MG is a sexually transmitted organism that is associated with symptoms of urethritis in men. Have we fulfilled Koch's postulates that MG is an etiologic agent for clinical symptoms in women? Initial, cross-sectional and observational data are conflicting. Animal models support biologic plausibility for inflammation and tissue damage. ${ }^{16,17}$ We await treatment data to show that symptoms or signs that are detected when the organism is present are shown to resolve after treatment. In addition, longitudinal data using various detection methods are needed to ascertain whether infection detected by recovery of viable organisms 
is different from colonization or transient positivity by nucleic acid amplification testing (NAAT).

\section{Conclusions}

M. genitalium is a highly prevalent STI in sexually active female adolescents and is strongly associated with current CT infection. We did not identify any clinical signs or symptoms that were significantly associated with MG infection. Some indicators such as positive CT and recent sexual contact may be reasons to obtain MG testing if it is available. The transcription-mediated amplification assay used in this study is sensitive and reliable but not widely available, and it may be prohibitively expensive for many clinicians. Accessible diagnostic methods will facilitate longitudinal studies that can accurately assess the role of MG as an etiology of genitourinary symptoms or other adverse outcomes such as pelvic inflammatory disease or acquisition of HIV.

\section{Acknowledgments}

Grace Kim, Stephanie Schubert, and Jennifer Bishop performed subject recruitment and data management. We thank Melissa Miller and John Schmitz for accommodating research testing in the McLendon Clinical Laboratories at UNC Hospitals.

J.S.H. has received unrestricted research funds, in-kind test kits, and speaker's honoraria from Genzyme Diagnostics. M.M.H. has received research funds for test evaluation, and in-kind test kits from Gen-Probe. Authors J.E.M., J.L.R., J.A.K., and K.D.R. report no conflict. Supported by the National Institutes of Health/National Institute for Allergy and Infectious Disease grant 5K23AI63182 (Huppert, PI). Dr. Hobbs and Ms. Rich were supported by NIH/NIAID grant U19-AI-31496. Kits and reagents were provided by Genzyme Diagnostics and Gen-Probe. No funding entity participated in the design or conduct of the study, in the collection, analysis, and interpretation of the data, or in the preparation, review, or approval of the manuscript.

\section{References}

1. Landers DV, Wiesenfeld HC, Heine RP, Krohn MA, Hillier SL. Predictive value of the clinical diagnosis of lower genital tract infection in women. Am J Obstet Gynecol. 2004; 190:1004-1010. [PubMed: 15118630]

2. Totten PA, Schwartz MA, Sjostrom KE, Kenny GE, Handsfield HH, Weiss JB, Whittington WL. Association of Mycoplasma genitalium with nongonococcal urethritis in heterosexual men. J Infect Dis. 2001; 183:269-276. [PubMed: 11120932]

3. Manhart LE, Critchlow CW, Holmes KK, Dutro SM, Eschenbach DA, Stevens CE, Totten PA. Mucopurulent cervicitis and Mycoplasma genitalium. J Infect Dis. 2003; 187:650-657. [PubMed: 12599082]

4. Korte JE, Baseman JB, Cagle MP, Herrera C, Piper JM, Holden AE, Perdue ST, Champion JD, Shain RN. Cervicitis and genitourinary symptoms in women culture positive for Mycoplasma genitalium. Am J Reprod Immunol. 2006; 55:265-275. [PubMed: 16533338]

5. Cohen CR, Nosek M, Meier A, Astete SG, Iverson-Cabral S, Mugo NR, Totten PA. Mycoplasma genitalium infection and persistence in a cohort of female sex workers in Nairobi, Kenya. Sex Transm Dis. 2007; 34:274-279. [PubMed: 16940898]

6. Tosh AK, Van Der Pol B, Fortenberry JD, Williams JA, Katz BP, Batteiger BE, Orr DP. Mycoplasma genitalium among adolescent women and their partners. J Adolesc Health. 2007; 40:412-417. [PubMed: 17448398]

7. Wroblewski JK, Manhart LE, Dickey KA, Hudspeth MK, Totten PA. Comparison of transcriptionmediated amplification and PCR assay results for various genital specimen types for detection of Mycoplasma genitalium. J Clin Microbiol. 2006; 44:3306-3312. [PubMed: 16954265]

8. Huppert JS, Mortensen JE, Reed JL, Kahn JA, Rich KD, Miller WC, Hobbs MM. Rapid antigen testing compares favorably with transcription- mediated amplification assay for the detection of Trichomonas vaginalis in young women. Clin Infect Dis. 2007; 45:194-198. [PubMed: 17578778] 
9. Hardick J, Giles J, Hardick A, Hsieh YH, Quinn T, Gaydos C. Performance of the gen-probe transcription-mediated [corrected] amplification research assay compared to that of a multitarget realtime PCR for Mycoplasma genitalium detection. J Clin Microbiol. 2006; 44:1236-1240. [PubMed: 16597844]

10. Anagrius C, Lore B, Jensen JS. Mycoplasma genitalium: Prevalence, clinical significance, and transmission. Sex Transm Infect. 2005; 81:458-462. [PubMed: 16326846]

11. Geisler WM, Yu S, Venglarik M, Schwebke JR. Vaginal leucocyte counts in women with bacterial vaginosis: Relation to vaginal and cervical infections. Sex Transm Infect. 2004; 80:401-405. [PubMed: 15459411]

12. Hakakha MM, Davis J, Korst LM, Silverman NS. Leukorrhea and bacterial vaginosis as in-office predictors of cervical infection in high-risk women. Obstet Gynecol. 2002; 100:808-812. [PubMed: 12383553]

13. Pepin J, Labbe AC, Khonde N, Deslandes S, Alary M, Dzokoto A, Asamoah-Adu C, Meda H, Frost E. Mycoplasma genitalium: An organism commonly associated with cervicitis among west African sex workers. Sex Transm Infect. 2005; 81:67-72. [PubMed: 15681727]

14. Falk L, Fredlund H, Jensen JS. Signs and symptoms of urethritis and cervicitis among women with or without Mycoplasma genitalium or Chlamydia trachomatis infection. Sex Transm Infect. 2005; 81:73-78. [PubMed: 15681728]

15. Piper JM, Korte JE, Holden AE, Shain RN, Perdue S, Champion JD, Newton ER. Development of composite symptom variables for quantitative analysis of genitourinary symptomatology in women. Int J STD AIDS. 2005; 16:128-132. [PubMed: 15807940]

16. Cohen CR, Manhart LE, Bukusi EA, Astete S, Brunham RC, Holmes KK, Sinei SK, Bwayo JJ, Totten PA. Association between Mycoplasma genitalium and acute endometritis. Lancet. 2002; 359:765-766. [PubMed: 11888591]

17. Taylor-Robinson D, Furr PM, Tully JG, Barile MF, Moller BR. Animal models of Mycoplasma genitalium urogenital infection. Isr J Med Sci. 1987; 23:561-564. [PubMed: 3667225] 


\section{TABLE 1}

Subject Demographic and History Characteristics Stratified by the Presence or Absence of M. genitalium

\begin{tabular}{lrrrl}
\hline & $\begin{array}{r}\text { Total } \\
(\mathbf{N = 3 3 1})\end{array}$ & $\begin{array}{r}\text { MG Positive } \\
(\mathbf{N = 7 4 )}\end{array}$ & $\begin{array}{r}\text { MG Negative } \\
(\mathbf{N = 2 5 7})\end{array}$ & $\boldsymbol{P}^{*}$ \\
\hline Age, 18-21 yr & $143(43.2)$ & $34(45.9)$ & $109(42.4)$ & 0.59 \\
Age, 14-7 yr & $188(56.8)$ & $40(54.1)$ & $148(57.6)$ & \\
Black race & $272(82.2)$ & $63(85.1)$ & $209(81.3)$ & 0.45 \\
Site of care = emergency department & $187(56.5)$ & $44(59.5)$ & $143(55.6)$ & 0.56 \\
Condom used at last sexual contact & $159(48.0)$ & $37(50.0)$ & $122(47.5)$ & 0.70 \\
Hormonal contraception used at last sexual contact & $116(35.0)$ & $19(25.7)$ & $97(37.7)$ & 0.055 \\
Self report of any prior STI & $206(62.2)$ & $47(63.5)$ & $159(61.9)$ & 0.79 \\
$>1$ sexual partner in last 3 mo & $86(25.9)$ & $26(35.1)$ & $60(23.4)$ & 0.04 \\
Recent sexual contact ( $\leq \mathrm{d})$ & $153(46.2)$ & $43(58.1)$ & $110(42.8)$ & 0.02 \\
New sexual partner in the last 3 mo & $100(30.2)$ & $21(28.4)$ & $79(30.7)$ & 0.69 \\
Low risk ( $\leq$ partner in last 3 mo and no new partners) & $198(59.8)$ & $41(55.4)$ & $157(61.2)$ & 0.38 \\
\hline
\end{tabular}

Data are displayed as number (column \%).

${ }^{*}$ value determined by $\mathrm{x}^{2}$ tests of significance.

MG indicates $M$. genitalium; STI = sexually transmitted infection. 
TABLE 2

Subject Symptoms, Signs, and Coinfections Stratified by Presence or Absence of M. genitalium

\begin{tabular}{|c|c|c|c|c|}
\hline & Total & MG Positive & MG Negative & $P^{*}$ \\
\hline Symptoms reported by subject & $\mathrm{N}=331$ & $\mathrm{~N}=74$ & $\mathrm{~N}=257$ & \\
\hline Vaginal discharge & $175(51.9)$ & $39(52.7)$ & $136(52.9)$ & 0.97 \\
\hline Vaginal itching & $107(32.3)$ & $20(27.0)$ & $87(33.8)$ & 0.27 \\
\hline Any vaginal symptoms & $211(63.9)$ & $44(59.6)$ & $167(64.9)$ & 0.38 \\
\hline Dysuria & $63(19.0)$ & $9(12.2)$ & $54(21.0)$ & 0.09 \\
\hline Abnormal vaginal bleeding & $40(12.1)$ & $9(12.2)$ & $31(12.1)$ & 0.9 \\
\hline Pelvic pain & $176(53.2)$ & $42(56.8)$ & $134(52.2)$ & 0.48 \\
\hline Any genitourinary symptoms & $295(89)$ & $64(86.5)$ & $231(89.9)$ & 0.40 \\
\hline Signs noted by provider ${ }^{\dagger}$ & $\mathrm{N}=318$ & $\mathrm{~N}=71$ & $\mathrm{~N}=247$ & \\
\hline Abnormal vaginal discharge & $137(43.1)$ & $26(36.6)$ & $111(44.9)$ & 0.21 \\
\hline Mucopurulent cervicitis & $47(14.8)$ & $7(9.9)$ & $40(16.2)$ & 0.18 \\
\hline Friable cervix & $52(16.4)$ & $11(15.5)$ & $41(16.6)$ & 0.82 \\
\hline Clinical cervicitis & $87(27.4)$ & $17(23.9)$ & $70(28.3)$ & 0.46 \\
\hline Cervical motion tenderness & $39(12.3)$ & $6(8.4)$ & $33(13.4)$ & 0.27 \\
\hline Uterine tenderness & $22(6.9)$ & $2(2.8)$ & $20(8.1)$ & $0.18^{t^{t}}$ \\
\hline Adnexal tenderness & $48(15.1)$ & $7(9.9)$ & $41(16.6)$ & 0.16 \\
\hline Any pelvic tenderness & $73(23.0)$ & $12(16.9)$ & $61(24.7)$ & 0.17 \\
\hline Clue cells $>20 \%$ of epithelial cells/HPF $\mathcal{E}$ & $70(23.7)$ & $14(20.0)$ & $52(23.1)$ & 0.58 \\
\hline $\mathrm{WBC}>10 / \mathrm{HPF} / /$ & $66(22.5)$ & $13(19.7)$ & $36(15.9)$ & 0.46 \\
\hline \multicolumn{5}{|l|}{ Coinfections ( $\mathrm{n}=331$ unless noted) } \\
\hline Trichomoniasis & $60(18.2)$ & $18(24.3)$ & $42(16.4)$ & 0.12 \\
\hline Chlamydia II & $79(24.4)$ & $27(37.5)$ & $52(20.6)$ & 0.003 \\
\hline Gonorrhea $^{\#}$ & $35(10.7)$ & $10(13.9)$ & $25(9.8)$ & 0.33 \\
\hline Either chlamydia or gonorrhea ${ }^{* *}$ & $97(30.0)$ & $31(43.6)$ & $66(26.2)$ & 0.005 \\
\hline Any STI & $129(39.0)$ & $40(54.0)$ & 89 (34.6) & 0.003 \\
\hline
\end{tabular}

Data are displayed as number $(\%)$.

$\mathrm{x}^{2}$ test, except as noted.

Missing for $3 \mathrm{MG}+$ and $10 \mathrm{MG}-$ subjects, total $=318$.

${ }^{*}$ Fisher exact test.

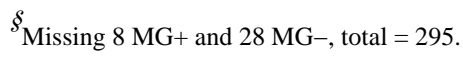

//Missing 8 MG+ and 30 MG-, total $=293$.

II Missing $2 \mathrm{MG}+$ and $5 \mathrm{MG}-$, total $=324$.

\# Missing 2 MG+ and 3 MG-, total $=326$.

**

Missing $3 \mathrm{MG}+$ and $5 \mathrm{MG}-$, total $=323$ 
MG indicates M. genitalium; $\mathrm{WBC}=$ white blood cells seen on vaginal wet mount or gram stain; HPF =high-powered field; STI = sexually transmitted infection with chlamydia, gonorrhea, or trichomoniasis. 


\section{TABLE 3}

Associations of Significant Variables With M. genitalium Infection in Female Adolescents Predicted by Logistic Regression, $\mathrm{N}=324$

\begin{tabular}{|c|c|c|c|c|}
\hline & \multicolumn{2}{|c|}{$\begin{array}{l}\text { Simple Logistic } \\
\text { Regression }\end{array}$} & \multicolumn{2}{|c|}{$\begin{array}{l}\text { Multiple Logistic } \\
\text { Regression Model }\end{array}$} \\
\hline & OR & $95 \% \mathrm{CI}$ & AOR & $95 \% \mathrm{CI}$ \\
\hline Chlamydial infection & 2.3 & $1.3-4.1$ & 2.5 & $1.4-4.4$ \\
\hline Recent sexual contact ( $\leq 7 \mathrm{~d}$ ) & 1.8 & $1.1-3.1$ & 2.0 & $1.1-3.2$ \\
\hline Dysuria & 0.52 & $0.24-1.1$ & 0.44 & $0.20-0.96$ \\
\hline Hormonal contraception & 0.59 & $0.33-1.1$ & 0.55 & $0.3-1.0$ \\
\hline$>1$ partners in last $3 \mathrm{mo}$ & 1.8 & $1.0-3.1$ & & \\
\hline
\end{tabular}

* Adjusted for all listed variables; the variable ">1 partners in last 3 mo" was highly correlated with hormonal contraception and did not reach significance in the final model.

OR indicates odds ratio; $\mathrm{CI}=$ confidence interval; $\mathrm{AOR}=$ adjusted odds ratio. 